\title{
ON PARTICLE FILTERING FOR DIGITAL COMMUNICATIONS
}

\author{
Tanya Bertozzi ${ }^{*}{ }^{*}$, Didier Le Ruyet ${ }^{\dagger}$, Gilles Rigal ${ }^{*}$ and Han Vu-Thien ${ }^{\dagger}$ \\ *DIGINEXT, 45 Impasse de la Draille, 13857 Aix en Provence Cedex 3, France, Email: bertozzi@ diginext.fr \\ ${ }^{\dagger}$ CNAM, 292 rue Saint Martin, 75141 Paris Cedex 3, France
}

\begin{abstract}
In this paper we analyze the problem of the joint channel-data estimation in fast fading channels. We propose a hybrid structure which respectively associates the Kalman filter and particle filtering for the channel and data estimation. We compare this solution with the classical reduced complexity methods. We show that the application of particle filtering to the discrete state space of the data leads to an approach similar to the T algorithm. Hence, this method cannot improve the trade-off between performance and computational complexity of the classical solutions. We conclude that it is preferable to use particle filtering for the joint estimation of discrete and continuous parameters.
\end{abstract}

\section{INTRODUCTION}

Particle Filtering (PF) or Sequential Monte Carlo (SMC) methods [1] represent the most powerful approach for the sequential estimation of the hidden state of a nonlinear dynamic model. The solution to this problem depends on the knowledge of the Posterior Probability Density (PPD) of the hidden state given the observations. Except in a few special cases, it is impossible to calculate analytically a sequential expression of this PPD. It is necessary to adopt numerical approximations. The SMC methods allow to approximate iteratively the PPD of the hidden state by weighted points or particles which evolve in the state space. Therefore, these methods provide a discrete approximation of the continuous space of the hidden state.

The first main applications of the SMC methods was target tracking. More recently, these techniques have been applied to solve different communication problems. In this paper, we will focus on the joint data-channel estimation in multipath fading channels. We will propose to use the SMC techniques for the estimation of the information sequence and the Kalman Filter (KF) for the estimation of the channel coefficients along each estimated data sequence. In this case, the state space is discrete and unlike the Viterbi Algorithm (VA), the SMC methods can reduce the computational complexity of the detector exploring only a subset of possible data sequences with a fixed number of particles. Hence, we will compare the SMC methods with the classical reduced complexity algorithms of the VA, such as the Per-Survivor Processing (PSP) algorithm [2, 3], the $\mathrm{M}$ algorithm [4] and the $\mathrm{T}$ algorithm [5].

This paper is organized as follows. In Section 2, we will introduce the system model. Then in Section 3, we will describe the detector based on the SMC methods. In Section 4, we will review the classical reduced complexity algorithms of the VA. Finally, simulation results are given in Section 5.

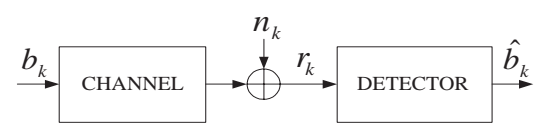

Fig. 1. Discrete-time equivalent lowpass transmission system model.

\section{SYSTEM MODEL}

Fig. 1 shows the discrete-time equivalent lowpass transmission model considered in this paper. Let us assume that the transmitted information is carried by a binary antipodal signal. The generalization to more complex modulations is straightforward. The data sequence is composed of independent and identically distributed (i.i.d.) bits. The information bits are organized into frames composed of a preamble of known bits used in the estimation of the Channel Impulse Response (CIR), a block of information bits and a tail of known bits for properly terminating the trellis. The discrete-time channel model is represented by a symbol-spaced $\mathrm{Fi}$ nite Impulse Response (FIR) filter. The CIR coefficients $\left\{f_{k, l}\right\}_{l=0}^{L}$ where $L$ indicates the overall channel memory, are unknown at the reception stage. We assume that the time variations of the CIR coefficients during the data frame are important and therefore, the channel estimation provided using the preamble is insufficient. It represents only an initialization of the CIR coefficient estimates, which must be tracked even in the data frame. The matrix model of the received signal at the input of the detector is given by:

$$
\mathbf{r}_{k}=\mathbf{B} \cdot \mathbf{F}_{k}+\mathbf{n}_{k}
$$

where:

$$
\begin{aligned}
& \mathbf{r}_{k}=\left[\operatorname{Re}\left\{r_{k}\right\} \operatorname{Im}\left\{r_{k}\right\}\right], \\
& \mathbf{B}=\left[\begin{array}{ll}
b_{k} & b_{k-1} \ldots b_{k-L}
\end{array}\right]=\left[\begin{array}{ll}
b_{k} & \mathbf{B}_{k-1}
\end{array}\right], \\
& \mathbf{B}_{k-1}=\left[b_{k-1} \ldots b_{k-L}\right] \text {, } \\
& \mathbf{F}_{k}=\left[\begin{array}{cc}
\operatorname{Re}\left\{f_{k, 0}\right\} & \operatorname{Im}\left\{f_{k, 0}\right\} \\
\vdots & \vdots \\
\operatorname{Re}\left\{f_{k, L}\right\} & \operatorname{Im}\left\{f_{k, L}\right\}
\end{array}\right], \\
& \mathbf{n}_{k}=\left[\operatorname{Re}\left\{n_{k}\right\} \operatorname{Im}\left\{n_{k}\right\}\right] \text {. }
\end{aligned}
$$

The vector $\mathbf{n}_{k}$ is a discrete-time complex AWGN with zero mean, scalar variance $\sigma_{n}^{2}$ and independent real and imaginary components. 


\section{PARTICLE FILTERING FOR THE JOINT DATA-CHANNEL ESTIMATION}

In [6] we have proposed to apply the SMC methods for the joint estimation of the CIR and the data sequence in fast fading conditions. In this section, we review this algorithm as the application of the SMC techniques to a discrete state space.

The optimal filtering problem involves the estimation of the hidden state $x_{t}$ of a system at a time $t$ using a sequence $y_{1: t}=$ $\left\{y_{1}, \cdots, y_{t}\right\}$ of noisy measurements made on the system. In order to solve this problem two models are required. Firstly, a model describing the evolution of the hidden state with time (state model) and secondly, a model relating the noisy observations to the state (observation model). In the Bayesian approach, the estimation of the hidden state is obtained from the PPD $p\left(x_{0: t} \mid y_{1: t}\right)$, where $x_{0: t}$ is the state sequence from 0 to $t$. In digital communication systems, the observations arrive sequentially in time and it is of interest to update at each instant the estimation of the hidden state. Hence, it is necessary to calculate the PPD recursively in time. Except in a few special cases, including linear Gaussian state space models and hidden finite-state space Markov chains, we cannot analytically solve this problem. The SMC methods allow to give a discrete approximation of the PPD which can be updated at each instant by evolving the particles in time. In general, the PPD is continuous and the technique to provide samples (particles) of this distribution is the Sequential Importance Sampling (SIS) method [7].

The detection of a data sequence in fading conditions presented in Section 2 can be interpreted as a Bayesian filtering problem, where the hidden state is represented by the sequence of information bits $B_{1: K}=\left\{b_{k} ; k=1, \cdots, K\right\}$ composed of $K$ bits and the sequence of the time-varying CIR coefficients $\mathbf{F}=\left\{\mathbf{F}_{k} ; k=\right.$ $1, \cdots, K\}$. The observation model is described by (1). We consider that the CIR coefficients are represented by a first-order $\mathrm{Au}$ toRegressive (AR) process, which corresponds to a Rayleigh uncorrelated scattering model:

$$
\mathbf{F}_{k}=\mathbf{A} \mathbf{F}_{k-1}+\mathbf{W}_{k} \quad \text { for } \quad k=1, \cdots, K,
$$

where:

$$
\begin{aligned}
\mathbf{A} & =\operatorname{diag}\left[\alpha_{0}, \cdots, \alpha_{L}\right] \quad \text { and } \\
\mathbf{W}_{k}= & {\left[\begin{array}{cc}
\operatorname{Re}\left\{w_{k, 0}\right\} & \operatorname{Im}\left\{w_{k, 0}\right\} \\
\vdots & \vdots \\
\operatorname{Re}\left\{w_{k, L}\right\} & \operatorname{Im}\left\{w_{k, L}\right\}
\end{array}\right] . }
\end{aligned}
$$

$\mathbf{W}_{k}$ is a complex discrete-time AWGN with zero-mean and covariance represented by the $(L+1) \times(L+1)$ matrix $\mathbf{Q}$. Since we have no a priori information on the speed of the time variations of the CIR coefficients, we will assume that $\mathbf{A}$ is an identity matrix. We can note that the state model (2) and the observation model (1) are linear and Gaussian for the channel. Hence, the optimal solution for the estimation of the CIR coefficients is the KF.

For the detection of the data sequence, we can use a Maximum Likelihood (ML) detector [8]. Given the sequence of received samples

$$
R_{1: K}=\left\{\mathbf{r}_{k} ; k=1, \cdots, K\right\},
$$

the ML detector selects, conditionally to the knowledge of the CIR coefficients, the sequence through the trellis that maximizes the

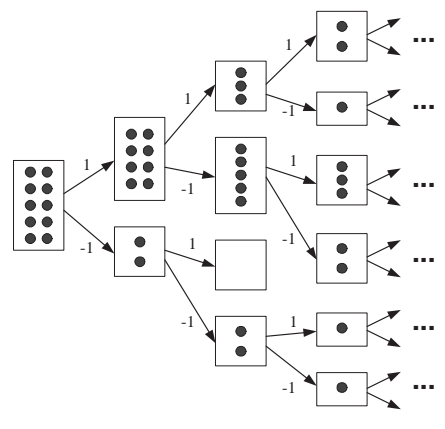

Fig. 2. The particle tree of the SIS algorithm.

following probability:

$$
\hat{B}_{1: K}=\arg \max _{B_{1: K}} p\left(B_{1: K} \mid R_{1: K}, \hat{\mathbf{F}}\right),
$$

where

$$
\hat{\mathbf{F}}=\left\{\hat{\mathbf{F}}_{k \mid k-1} ; k=1, \cdots, K\right\}
$$

is the sequence of the CIR coefficients estimates and $\hat{\mathbf{F}}_{k \mid k-1}$ the estimate of the CIR coefficients at time $k$ knowing the received samples until time $k-1$. The main drawback of the ML detector is that its computational complexity increases exponentially with the channel memory. In order to reduce the computational complexity of the detector, we propose in this paper to use the SIS algorithm. This algorithm, applied to a discrete state space, belongs to the family of tree-search algorithms. We will see that the leaves of the tree are simply groups of particles, as depicted in Fig. 2. We describe the derivation of the SIS algorithm for a discrete state space. The aim of this method is to approximate recursively in time the PPD in (4) with weighted particles:

$$
p\left(B_{1: K} \mid R_{1: K}, \hat{\mathbf{F}}\right) \approx \sum_{i=1}^{N} \tilde{w}_{K}^{(i)} \delta\left(x_{K}-x_{K}^{(i)}\right) \cdots \delta\left(x_{1}-x_{1}^{(i)}\right)
$$

where $N$ is the number of particles, $\tilde{w}_{K}^{(i)}$ is the normalized importance weight at time $K$ associated with the particle $i$ and $\delta\left(x_{k}-\right.$ $\left.x_{k}^{(i)}\right)$ denotes the Dirac delta centered in $x_{k}=x_{k}^{(i)}$ for $k=$ $1, \cdots, K$. Initially, the particles are in the same state, composed of the $L$ last bits of the preamble. This state is the root of the tree. The state space is already discretized and then, the particles can only be moved towards the possible states of the system. The particles explore the state space in groups, which represent the leaves of a tree. In fact, for each group at a time $k-1$ two values are possible for the current bit $b_{k}:+1$ and -1 . Hence, the particles will be divided in two groups. In the conventional SIS algorithm, the time evolution of the particles is achieved with an importance sampling distribution. For the transition from time $k-1$ to time $k$, the particles are drawn according to the importance function $\pi\left(b_{k}^{(i)} \mid B_{1: k-1}^{(i)}, R_{1: k}\right)$. We choose the optimal importance function which minimizes the variance of the importance weights of the particles [7]:

$$
\pi\left(b_{k}^{(i)} \mid B_{1: k-1}^{(i)}, R_{1: k}\right)=p\left(b_{k}^{(i)} \mid \mathbf{B}_{k-1}^{(i)}, \hat{\mathbf{F}}_{k \mid k-1}^{(i)}, \mathbf{r}_{k}\right),
$$

where $\mathbf{B}_{k-1}^{(i)}=\left[b_{k-1}^{(i)} \cdots b_{k-L}^{(i)}\right]$ is the term of InterSymbol Interference (ISI) due to the channel memory. In this case, the particles 


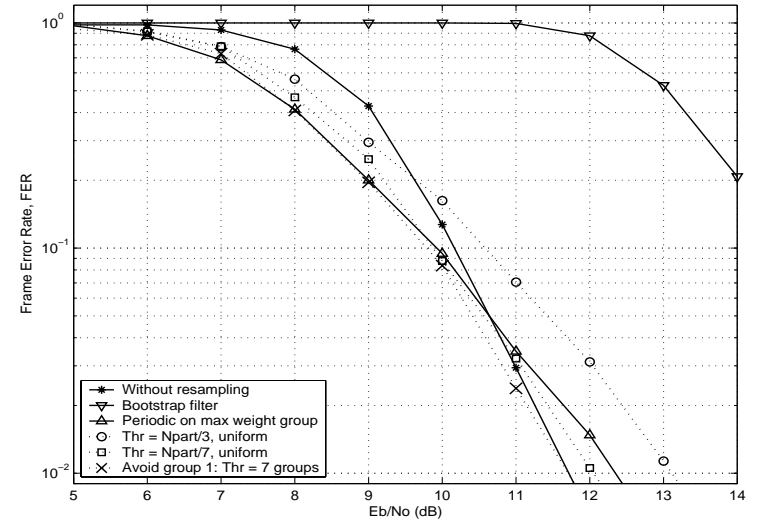

Fig. 3. FER versus Eb/No: Different resampling techniques for the PD with 20 particles.

cannot be drawn because the states are discrete. However, the particles of a group at time $k-1$ are divided proportionally to the importance function in two groups at time $k$. Owing to the indivisibility of the particles, it can occur that some groups are empty. The path associated to an empty group is eliminated. We calculate (6), assuming that the current bit $b_{k}=1$. The calculation for $b_{k}=-1$ is similar. Applying the Bayes theorem, the importance function assumes the form:

$$
\begin{gathered}
p\left(b_{k}^{(i)}=1 \mid \mathbf{B}_{k-1}^{(i)}, \hat{\mathbf{F}}_{k \mid k-1}^{(i)}, \mathbf{r}_{k}\right) \\
=\frac{p\left(\mathbf{r}_{k} \mid \mathbf{B}_{+}^{(i)}, \hat{\mathbf{F}}_{k \mid k-1}^{(i)}\right) p\left(b_{k}^{(i)}=1 \mid \mathbf{B}_{k-1}^{(i)}, \hat{\mathbf{F}}_{k \mid k-1}^{(i)}\right)}{\int p\left(\mathbf{r}_{k} \mid \mathbf{B}^{(i)}, \hat{\mathbf{F}}_{k \mid k-1}^{(i)}\right) p\left(b_{k}^{(i)} \mid \mathbf{B}_{k-1}^{(i)}, \hat{\mathbf{F}}_{k \mid k-1}^{(i)}\right)}
\end{gathered}
$$

where $\mathbf{B}_{+}^{(i)}=\left[\begin{array}{ll}b_{k}^{(i)}=1 & \mathbf{B}_{k-1}^{(i)}\end{array}\right]$. Since the information bits are i.i.d., we can write:

$$
p\left(b_{k}^{(i)}=1 \mid \mathbf{B}_{k-1}^{(i)}, \hat{\mathbf{F}}_{k \mid k-1}^{(i)}\right)=\frac{1}{2}
$$

Therefore, (7) becomes:

$$
\begin{gathered}
p\left(b_{k}^{(i)}=1 \mid \mathbf{B}_{k-1}^{(i)}, \hat{\mathbf{F}}_{k \mid k-1}^{(i)}, \mathbf{r}_{k}\right) \\
=\frac{p\left(\mathbf{r}_{k} \mid \mathbf{B}_{+}^{(i)}, \hat{\mathbf{F}}_{k \mid k-1}^{(i)}\right)}{p\left(\mathbf{r}_{k} \mid \mathbf{B}_{+}^{(i)}, \hat{\mathbf{F}}_{k \mid k-1}^{(i)}\right)+p\left(\mathbf{r}_{k} \mid \mathbf{B}_{-}^{(i)}, \hat{\mathbf{F}}_{k \mid k-1}^{(i)}\right)} .
\end{gathered}
$$

We observe that the probability densities $p\left(\mathbf{r}_{k} \mid \mathbf{B}_{+}^{(i)}, \hat{\mathbf{F}}_{k \mid k-1}^{(i)}\right)$ and $p\left(\mathbf{r}_{k} \mid \mathbf{B}_{-}^{(i)}, \hat{\mathbf{F}}_{k \mid k-1}^{(i)}\right)$ are Gaussian with mean $\mathbf{B}^{(i)} \hat{\mathbf{F}}_{k \mid k-1}^{(i)}$ and scalar variance $\mathbf{B}^{(i)} \tilde{\mathbf{P}}_{k \mid k-1} \mathbf{B}^{(i) T}+\sigma_{n}^{2}$, where we have omitted the subindex + or - and $\tilde{\mathbf{P}}_{k \mid k-1}=E\left\{\tilde{\mathbf{F}}_{k \mid k-1}^{(i)} \tilde{\mathbf{F}}_{k \mid k-1}^{(i) T}\right\}$ is the covariance matrix of the estimation error $\tilde{\mathbf{F}}_{k \mid k-1}^{(i)}=\mathbf{F}_{k}-\hat{\mathbf{F}}_{k \mid k-1}^{(i)}$ provided by the KF. Using the optimal importance function, the importance weights are given by [7]:

$$
\begin{aligned}
w_{k}^{(i)} & =w_{k-1}^{(i)} p\left(\mathbf{r}_{k} \mid \mathbf{B}_{k-1}^{(i)}, \hat{\mathbf{F}}_{k \mid k-1}^{(i)}\right) \\
& =p\left(\mathbf{r}_{k} \mid \mathbf{B}_{+}^{(i)}, \hat{\mathbf{F}}_{k \mid k-1}^{(i)}\right)+p\left(\mathbf{r}_{k} \mid \mathbf{B}_{-}^{(i)}, \hat{\mathbf{F}}_{k \mid k-1}^{(i)}\right)
\end{aligned}
$$

and the normalized importance weights in (5) are calculated by:

$$
\tilde{w}_{k}^{(i)}=\frac{w_{k}^{(i)}}{\sum_{j=1}^{N} w_{k}^{(j)}} .
$$

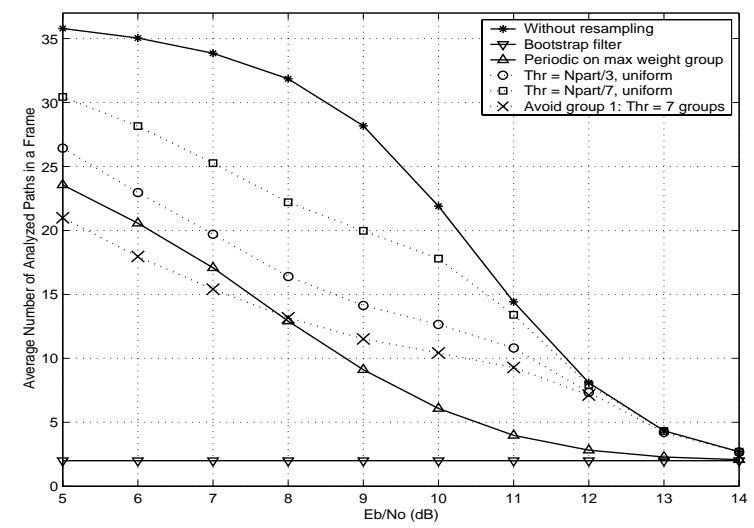

Fig. 4. Complexity versus Eb/No: Different resampling techniques for the PD with 20 particles.

At the end of the frame, we decide that the best path is the transmitted data sequence. The best path is the path associated with the group of particles with maximum weight. The weight of a group is given by the sum of the weights of the particles in the group.

The SIS algorithm applied to a continuous state space presents a degeneracy phenomenon when the time increases. After a few iterations of the algorithm, only one particle has a normalized weight almost equal to 1 and the other weights are very close to zero. Therefore, a large computational effort is devoted to updating paths without almost no contribution to the final estimate. In order to avoid this behavior, different strategies of resampling of the particles have been developed [1]. When we apply the SIS algorithm to a discrete state space, we can observe the same degeneracy phenomenon: due to the indivisibility of the particles, the SIS algorithm cannot find the most likely path in the complete tree. At low Signal-to-Noise Ratio (SNR) the particle tree grows until each group contains only one particle, as shown in Fig. 2. From this time, each particle can only explore the most likely of the two new paths. However, if the rejected path was the correct one, the Particle Detector (PD) definitely loses it. In consequence, in order to avoid the case where each group contains only one particle, we should apply a so called resampling phase. There exist different resampling solutions. A first solution is the bootstrap filter [9], where the particles are resampled at each time according to their importance weights. Another solution is to introduce a threshold that determines the time of resampling. This threshold parameter can be fixed or adapted according to the SNR. A last solution is a deterministic resampling which redistributes periodically the particles according to a predefined low.

\section{CLASSICAL REDUCED COMPLEXITY ALGORITHMS}

The classical reduced complexity algorithms are based either on the selection of a subset of the states in the code trellis, as in the PSP methods [2,3] or on the selection of a subset of the paths in the code trellis, as in the M algorithm [4] and in the T algorithm [5]. The PSP methods are a family of algorithms that compensate the uncertainties in the detector by correcting the transition metrics of the VA in a per-survivor way. In this paper, we will use the PSP algorithms in order to reduce the computational complexity of 


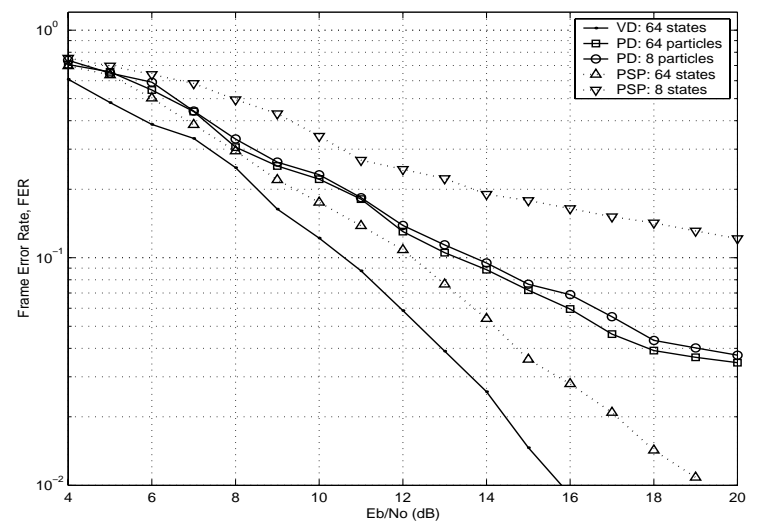

Fig. 5. FER versus Eb/No: Comparison PSP-PD.

the detector. In general, the $\mathrm{M}$ algorithm is described on a trellis and the $\mathrm{T}$ algorithm on a tree. From one iteration to the next one, the $\mathrm{M}$ algorithm keeps the $M$ best paths, where $M$ is less than the total number of states. On the other hand, the T algorithm keeps a variable number of paths depending on the threshold parameter $T$. If the relative likelihood of the considered path with respect to the likelihood of the best path is greater than $T$, the path is eliminated. Unlike the $\mathrm{M}$ algorithm which has a fixed complexity, the number of analyzed paths in the $\mathrm{T}$ algorithm depends on the time variations of the observation noise and changes during the data frame. Moreover, the computational complexity of the T algorithm is adapted according to the SNR. Generally, we also limit the number of survivor paths in order to avoid that at low SNR this number increases indefinitely. So when the number of survivor paths becomes greater than $S$, we will keep the $S$ best paths. For the three methods described above, we will estimate the channel along each path with the KF in a per survivor way [10].

\section{SIMULATION RESULTS}

In this section, we will compare the performance of PF with different resampling methods and the classical reduced complexity algorithms. The simulation results are given for the Global System for Mobile communications (GSM), where each frame is processed independently. The preamble is composed of 26 known bits, followed by a data block of 58 information bits. We assume that the received samples are described by the model (1).

Fig. 3 and 4 show the performance and the computational complexity of PF for different methods of resampling. In order to analyze the differences among these techniques, we have chosen a deterministic time-varying minimum-phase channel with memory equal to 7 and CIR coefficients given by:

$$
f_{k, l}=a_{l} e^{j 2 \pi f_{l} k T_{s}}
$$

for $l=0, \cdots, L$, where the normalized amplitudes

$$
\left[a_{0}, \ldots, a_{7}\right]=[0.56,0.49,0.42,0.35,0.28,0.21,0.14,0.07],
$$

the frequencies in $\mathrm{Hz}$

$$
\left[f_{d, 0}, \ldots, f_{d, 7}\right]=[10,20,30,40,50,60,70,80]
$$

and the sampling period $T_{s}$ is equal to the symbol interval $T=$ $3.69 \mu \mathrm{s}$. As explained in Section 3, we observe that it is necessary

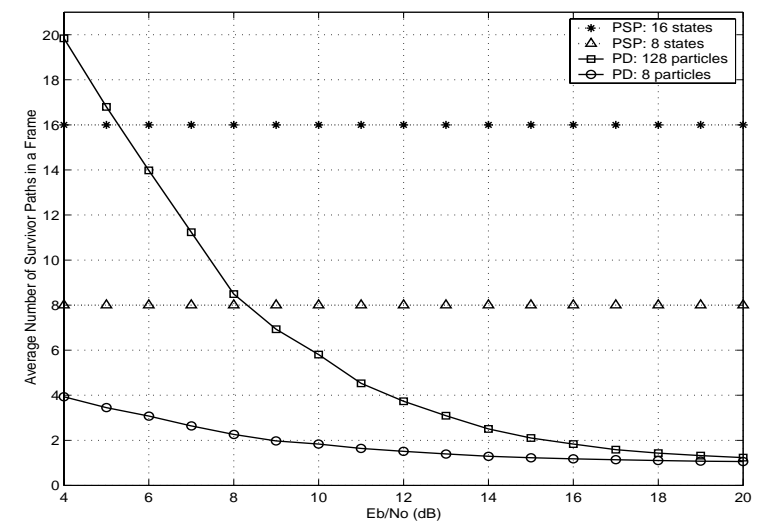

Fig. 6. Complexity versus Eb/No: Comparison PSP-PD.

to resample the particles at low SNR in order to avoid that each group contains only one particle. We cannot resample the particles at each time, as in the bootstrap filter solution, because we prevent the particles to explore more than two paths and the tree cannot grow. We have considered two other methods of resampling: deterministic and activated by a threshold parameter. For the first method, we have found that the best solution is to redistribute the particles with a period equal to the channel memory. At each period, the particles are moved into the group with maximum weight. At low SNR, the periodic resampling gives better performance than the solution without resampling. However, at high SNR the particles remain grouped, because the transition probabilities are very close to zero or one. Hence, in this case it is better not to resample the particles which explore only the more probable zones of the state space without loss of the best path. For the second method, we have considered the resampling procedure of the SIS algorithm [7]. If

$$
N_{e f f} \approx \frac{1}{\sum_{i=1}^{N} w_{k}^{(i) 2}}<N_{\text {thresh }},
$$

the particles are redistributed uniformly according to their importance weights. If we increase $N_{\text {thresh }}$ from $N / 3$ to $N / 7$, the performance at low SNR approaches the one with periodic resampling and at high SNR it is closer to the one without resampling. However, the computational complexity of this resampling technique is always higher than that of the periodic solution. In order to take the advantages of the previous resampling methods, we have chosen a threshold $N_{\text {one }}$ allowing to adapt the performance to the SNR. When the number of groups with one particle is greater than $N_{\text {one }}$, the particles are moved into the group with maximum weight. We conclude that this solution represents the best trade-off between performance and computational complexity.

Fig. 5 and 6 show the comparison of PF with the PSP detector for a 12-tap Hilly Terrain (HT) GSM channel, described in [11]. We have considered fast fading conditions with a Doppler frequency for each path equal to $200 \mathrm{~Hz}$. This corresponds to a vehicle speed of $240 \mathrm{~km} / \mathrm{h}$ for a $900 \mathrm{MHz}$ GSM system. The channel memory $L$ of a HT channel is equal to 6 . If we reduce the number of states in the PSP detector, we observe a frame error floor. This behavior is absent in the PD if we reduce the number of particles. Unlike the PSP detector, the computational complexity of the PD is adapted according to the SNR. As a consequence, for the same 


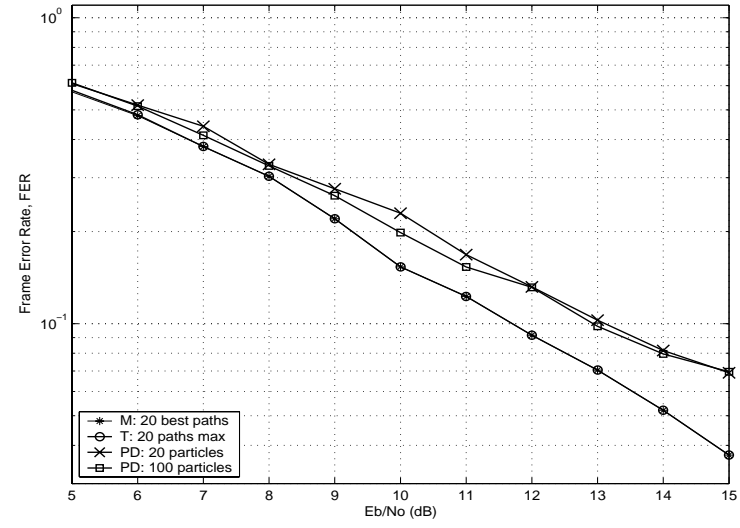

Fig. 7. FER versus Eb/No: Comparison $\mathrm{M}$ algorithm- $\mathrm{T}$ algorithmPD.

performance, except at low $E_{b} / N_{0}$, the complexity of the PD is always lower than the PSP detector. Hence, in long memory channel the PD presents a better trade-off between performance and computational complexity than the PSP detector.

In Fig. 7 and 8 we compare the PD with the $\mathrm{M}$ and $\mathrm{T}$ algorithms for a HT240 channel. The performance of the T algorithm coincides with that of the $\mathrm{M}$ algorithm. However the first one presents a lower complexity. The PD is similar to the $\mathrm{T}$ algorithm. Both algorithms have a computational complexity which changes during the data frame and which is adapted to the SNR. We have observed that the PF cannot improve the trade-off between performance and computational complexity provided by the $\mathrm{T}$ algorithm. The main difference between these solutions is the exploration of the state space. For the T algorithm, the rejection of the explored paths is related to a continuous variable: the difference between the likelihood of the best path and the likelihood of the analyzed path. For the PD, the rejection depends on a discrete quantity: the number of particles in a group. The particles represent a quantization of the likelihood of the T algorithm. Hence, by using indivisible entities (the particles), we lose some information contained in the likelihood. As a consequence, the PD cannot give better performance than that of the $\mathrm{T}$ algorithm.

\section{CONCLUSIONS}

In this paper, we have proposed a hybrid solution for the joint estimation of the data and the channel in fast fading conditions, based on PF for the detection of the information sequences and on the $\mathrm{KF}$ for the estimation of the CIR coefficients. While generally used for continuous state spaces, we have applied PF to the discrete state space of data. Then, we have compared this detector with the classical reduced complexity methods. To some extend, we have discovered a close version of the $\mathrm{T}$ algorithm when applying PF on a discrete state space. However, we have shown that, because of the indivisibility of the particles, PF cannot reaches the performance of the $\mathrm{T}$ algorithm for the same computational complexity.

Field of future researches will be the application of PF to digital communication problems where the unknown parameters consist not only of data but also of some continuous-valued parameters, such as for the joint delay-channel-data estimation in DSCDMA systems. In this case, PF can improve the performance

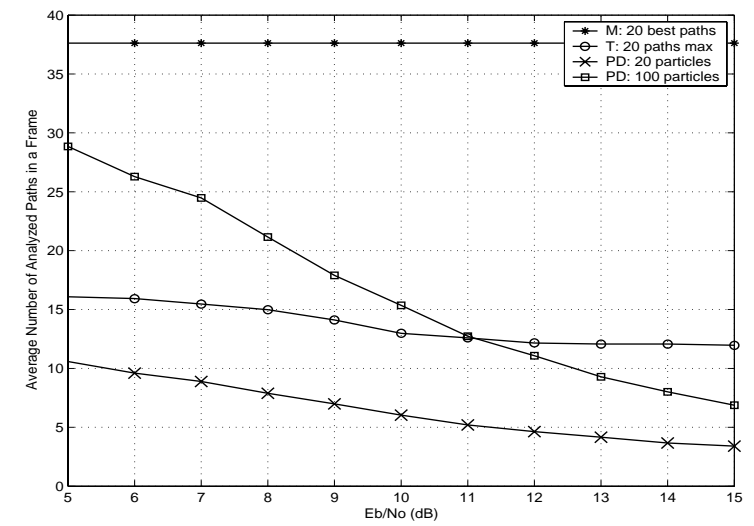

Fig. 8. Complexity versus Eb/No: Comparison $\mathrm{M}$ algorithm- $\mathrm{T}$ algorithm-PD.

obtained with the classical methods.

\section{REFERENCES}

[1] A. Doucet, J. F. G. de Freitas and N. J. Gordon, Sequential Monte Carlo methods in practice. New York: SpringerVerlag, 2001.

[2] M. V. Eyuboğlu and S. U. H. Qureshi, "Reduced-state sequence estimation with set partitioning and decision feedback,” IEEE Trans. on Com., Vol. 36, pp. 13-20, Jan. 1988.

[3] A. Duel-Hallen and C. Heegard, "Delayed decision-feedback sequence estimation," IEEE Trans. on Com., Vol. 37, pp. 428-436, May 1989.

[4] F. Jelinek and J. B. Anderson, "Instrumentable tree encoding of information sources," IEEE Trans. on Inf. Theory (Corresp.), Vol. 17, pp. 118, Jan. 1971.

[5] S. J. Simmons, "Breadth-first trellis decoding with adaptive effort," IEEE Trans. on Com., Vol. 38, pp. 3-12, Jan. 1990.

[6] T. Bertozzi, D. Le Ruyet, G. Rigal and H. Vu-Thien, "Joint data-channel estimation using the particle filtering on multipath fading channels," To appear in the Int. Conf. on Telecom. (ICT) Proceedings 2003, Feb. 2003.

[7] A. Doucet, S. Godsill and C. Andrieu, "On sequential Monte Carlo sampling methods for Bayesian filtering," Statistics and Computing, Vol. 10, No. 3, pp. 197-208, 2000.

[8] G. D. Forney, "Maximum likelihood sequence stimation of digital sequences in the presence of intersymbol interference," IEEE Trans. on Inf. Theory, Vol. 18, pp. 363-378, May 1972.

[9] N. J. Gordon, D. J. Salmond and A. F. M. Smith, "Novel approach to nonlinear/non-Gaussian Bayesian state estimation," IEE Proc., Vol. 140(2), pp. 107-113, 1993.

[10] C. -K. Tzou, R. Raheli and A. Polydoros, "Applications of Per-Survivor Processing to mobile digital communications," Proc. IEEE Globecom, Nov. 1993.

[11] GSM Recommandations 05.05 Version 6.3.0 Release 1997, "Digital cellular telecommunications system (Phase 2+); radio transmission and reception." 